\title{
Provimento aos cargos de diretores: efeitos em escolas públicas fluminenses
}

\author{
Sergio Gonçalves da Cunha
}

LAPOPE-UFRJ

\begin{abstract}
Resumo
Foi realizado um quase-experimento avaliando impactos das mudanças nas formas de provimento ao cargo de gestores de escolas fluminenses no trabalho docente e no desempenho discente, utilizando dados da Prova Brasil (2007 a 2015), Imputações Múltiplas para lidar com perdas amostrais, Análise Fatorial para criação de Índices de liderança dos diretores e o estimador Diferenças em Diferenças para captação de efeitos nas variáveis de interesse. Os resultados sugerem que não houve mudanças estatisticamente significativas no desempenho dos alunos e na percepção de liderança entre os docentes nas unidades investigadas em Macaé ao adotarem prova associada à eleição. Nas demais escolas tratadas, mudanças ocorreram no desempenho dos alunos em Língua Portuguesa e Matemática após a adoção das eleições.
\end{abstract}

Palavras-chave: Gestão Escolar; Liderança; Desempenho.

\begin{abstract}
Appointment to the positions of the school principals: effects in municipal public schools in Rio de Janeiro
\end{abstract}

A quasi-experimental design study was conducted assessing impacts of changes in the forms of appointment to the position of the school principals of schools in Rio de Janeiro in teaching work and student performance using data from Prova Brasil (2007-2015), multiple imputations to deal with case losses, Factor Analysis for the creation of a School Principals Leadership Index and the diff-in-diff estimator to observe the effects of variables of interest. The results suggest that there were no statistically significant changes in the performance of students and in the perception of the principals leadership by the teachers in the schools investigated in Macaé that started to adopt selection by test and election. In the other schools treated, changes occurred in the performance of students in Portuguese and Mathematics after the adoption of the elections.

Keywords: School Management; Leadership; Performance.

\section{Resumen}

\section{Admisión a los puestos de directores: efectos en escuelas públicas de Rio de Janeiro}

Se llevó a cabo un cuasi-experimento para evaluar los impactos de los cambios en las formas de admisión de directores de escuelas de Río de Janeiro en el trabajo docente y el rendimiento de los estudiantes, utilizando datos de Prova Brasil (2007 a 2015), Imputaciones múltiples para lidiar con pérdidas de muestras, Análisis Factorial para crear los índices de liderazgo de los directores y el estimador "diferencia en diferencias" para capturar los efectos en las variables de interés. Los resultados sugieren que no hubo cambios estadísticamente significativos en el desempeño de los estudiantes y la percepción de liderazgo entre los docentes en las unidades investigadas en Macaé al adoptar las pruebas asociadas 
a las elecciones. En las otras escuelas tratadas, se produjeron cambios en el rendimiento de los estudiantes en las asignaturas de lengua portuguesa y matemática después de la adopción de las elecciones. Palabras clave: Gestión Escolar; Liderazgo; Rendimento.

\section{Introdução}

A consulta pública à comunidade para a escolha dos diretores de escolas é defendida (presente em documentos oficiais e em diversos estudos na área para o contexto brasileiro) como uma importante diretriz da educação nacional. Mas seria essa diretriz um fim em si mesmo ou um meio efetivo para alcançar uma educação de qualidade? Estaria ela associada à maior aprendizagem e progressão dos alunos? Tal forma de provimento trata-se de um desmembramento do princípio da gestão democrática em expansão no Brasil há pelo menos três décadas. Algo semelhante parece ocorrer com outras formas de provimento ao cargo que, em certa medida, também se distanciam das historicamente recorrentes indicações políticas (como no caso dos modelos mistos, ou seja, que associam processos seletivos através de provas e eleições).

Seguem resultados de uma investigação sobre como mudanças nas formas de provimento ao cargo de diretores de escolas públicas municipais de ensino fundamental de cidades fluminenses alteraram a percepção dos professores sobre a liderança dos seus gestores e o desempenho acadêmico dos estudantes. Por meio de pesquisa quantitativa com desenho quase-experimental, investigou as seguintes questões: mudanças nas formas de provimento ao cargo de diretores de escolas podem alterar a forma na qual os docentes avaliam a sua liderança? Essas mesmas mudanças podem gerar impactos no desempenho dos estudantes?

Desde a década de 1980 no Brasil, os diretores são tomados como objetos de pesquisa considerando-se seus papéis desempenhados e/ou suas formas de ingresso nas instituições de ensino. Com isso, uma das diretrizes da gestão democrática que vêm ganhando força também como elemento de estudo é a forma de provimento ao cargo via consulta pública à comunidade (ALVES, 2007; LÜCK, 2009; 2011; ESQUINSANI, 2013). Como discussão internacional, a importância da figura dos diretores de escolas está centrada na relação entre características dos diretores, a sua liderança e o desempenho dos estudantes (DHUEY, SMITH, 2010; LEITHWOOD, JANTZI, 2000; LEITHWOOD, SUN, 2009). Por outro lado, mesmo com a ampliação das discussões sobre o tema, as novas diretrizes para a educação no Brasil sinalizam que a relação entre mecanismos de efetivação da gestão democrática (como a escolha de diretores) e qualidade da educação é tomada como dada, ou seja, a referida associação é defendida de maneira trivializada. Há escassas indicações consistentes no Brasil sobre a associação entre formas provimento e/ou exercício da gestão 
escolar, a liderança exercida (ou não) nas escolas e a qualidade da educação ofertada (desempenho discente). Entretanto, há estudos relacionados à eficácia escolar que já identificaram algumas características de liderança, capazes de promover maior eficácia e equidade na escola (OLIVEIRA, 2015; PENA, 2013; POLON, 2009; OLIVEIRA, CARVALHO, 2018). Internacionalmente podemos citar também como exemplo os estudos de Sammons (2008).

Cabe observar o que já sabemos, em especial no contexto brasileiro, sobre características da gestão escolar e do trabalho do gestor e sua relação com desempenho dos alunos, com ênfase em estudos que utilizam controles relacionados ao perfil do alunado e outras características pertinentes da escola. Estes estudos, em geral, têm desenho correlacional, utilizam dados secundários de avaliações externas e do Censo Escolar além de modelos de Regressão Multinível ${ }^{1}$. Entre as limitações observadas nota-se o fato de haver, por exemplo, poucas discussões sobre a qualidade dos dados secundários. Além disso, e da importância da gestão exercida com liderança, também há pesquisas que demonstraram uma associação negativa entre diretores eleitos e desempenho dos estudantes (SANTOS, SAMPAIO, SAMPAIO, 2014; BIONDI, FELICIO, 2008). Metodologicamente destaca-se a colaboração de Santos, Sampaio e Sampaio (2014) e Gremaud, Pazello e Maluf (2016) pois trata-se de quase-experimentos e com a utilização do método Diferença em Diferenças (diff-in-diff). Nesta última pesquisa reforça-se ainda que há poucos trabalhos no país envolvendo estudos sobre a figura do diretor escolar.

\section{O desenho da pesquisa}

Trata-se de um estudo com desenho quase experimental (CAMPBELL, STANLEY, 1963). As escolas envolvidas não puderam ser escolhidas aleatoriamente, logo, as amostras são classificadas como não probabilísticas (teóricas) e foram selecionadas em função dos documentos disponíveis dos municípios (legislação sobre formas de provimento ao cargo de diretores). Em resumo: iniciou-se com a leitura e interpretação dos documentos dos municípios, foram organizadas as informações sobre as formas de provimento ao cargo de diretores e desempenho dos estudantes, além da preparação e discussão das estatísticas descritivas para as variáveis dependentes e definição dos municípios e escolas para comporem os grupos de controle e tratamento nos diferentes bancos. Na sequência, foram realizadas análises de viés de seleção e além da discussão de seus resultados a partir da comparação entre os grupos de controle e tratamento (escolas dentro de um mesmo

\footnotetext{
${ }^{1}$ Ver mais detalhes sobre alguns desses estudos em Cunha (2019).
} 
banco de dados) e entre diferentes bancos. Para isso, além dos dados descritivos sobre os municípios e escolas, foram levados em conta resultados de testes Qui-quadrados (FIELD, 2009) para frequências considerando principalmente a variável "Complexidade_da_gestao”, a partir da comparação entre os grupos de escolas. No que diz respeito à variável de interesse/resultados correspondentes à liderança do diretor, recorreu-se às respostas dos professores aos questionários contextuais da Prova Brasil para elaboração de um índice de liderança, tal qual feito por Oliveira (2015), Oliveira e Waldhelm (2016) e Oliveira e Carvalho (2018) através da técnica de Análise Fatorial (ibidem) com redução a um fator. Em seguida, agregaram-se os índices ao nível das escolas que foram as unidades de análises em questão. O Gráfico apresenta um esquema geral do desenho de pesquisa utilizado.

Gráfico. Esquema geral do desenho de pesquisa para as análises realizadas.

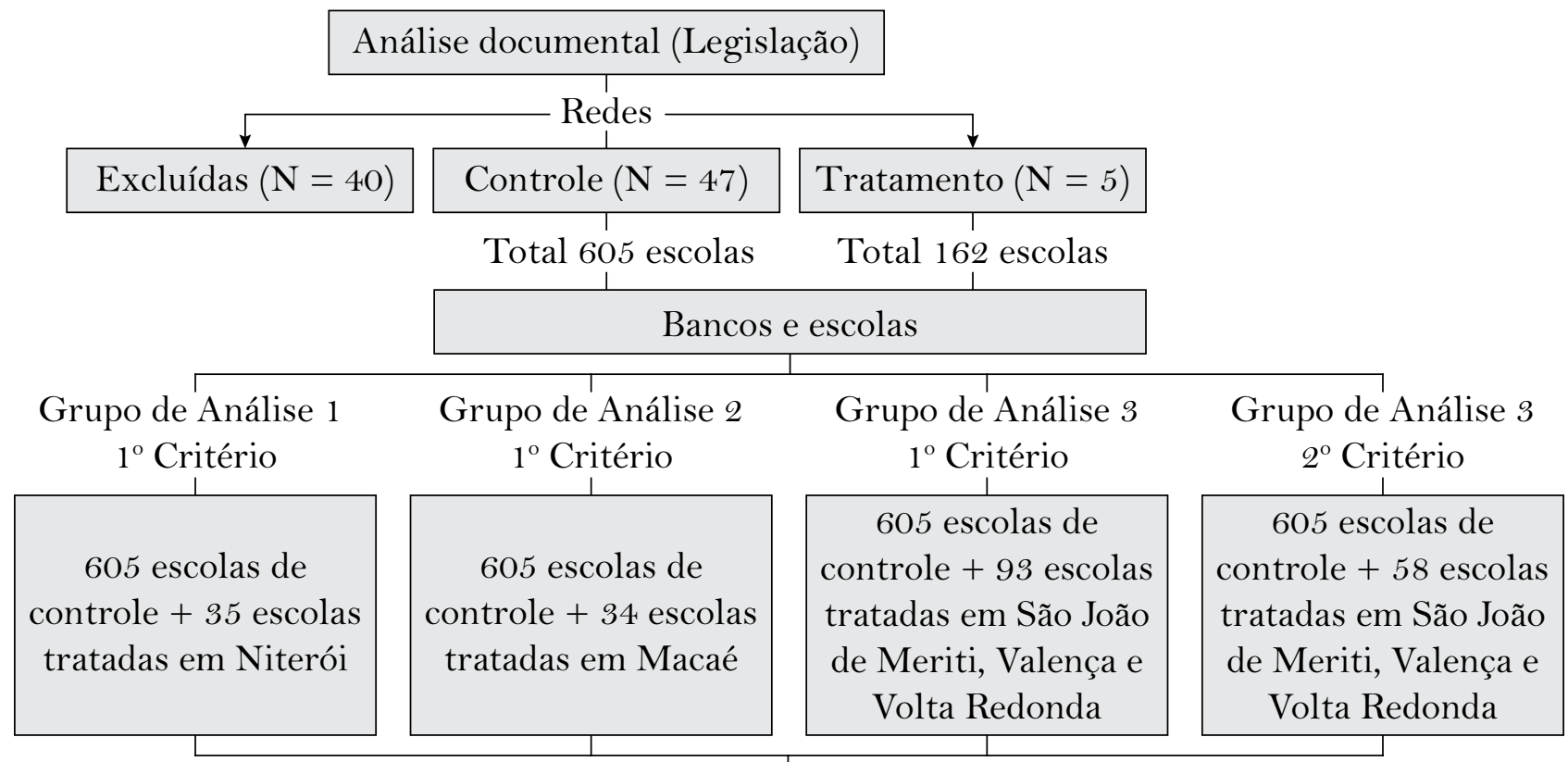

Variáveis dependentes e independentes: respostas dos professores (Análise Fatorial), desempenho das escolas, ano

de adoção do programa, programa e termo interativo

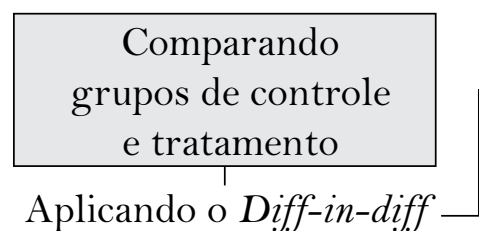

( $1^{\text {a }}$ etapa)

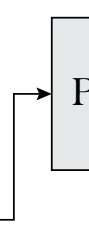

Proficiência média matemática Proficiência média língua portuguesa Índice de liderança do diretor Resultado do Diff-in-diff ( $1^{\text {a }}$ etapa)

Diferença em diferenças entre os grupos de cada banco

Coeficientes e significância

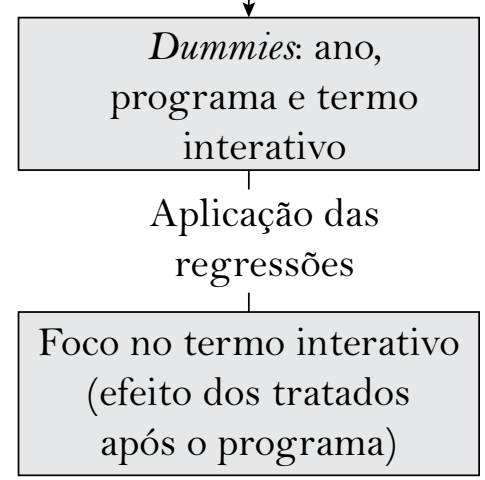

Dummies: ano, rograma e termo Aplicação das regressões (efeito dos tratados após o programa) 
O resumo com informações sobre as fontes, níveis de mensuração e caracterização das variáveis utilizadas para as análises seguem no Quadro 1.

Quadro 1. Variáveis utilizadas nas análises.

\begin{tabular}{|c|c|c|c|c|}
\hline $\begin{array}{l}\text { Variável e sua } \\
\text { condição nas } \\
\text { análises }\end{array}$ & Fontes & Тipo & $\begin{array}{c}\text { Nível de } \\
\text { mensuração }\end{array}$ & Características \\
\hline $\begin{array}{l}\text { Ano } \\
\text { (independente) }\end{array}$ & $\begin{array}{c}\text { Documentos sobre } \\
\text { manutenção ou } \\
\text { mudanças nas formas } \\
\text { de provimento ao cargo } \\
\text { de diretores (PME, } \\
\text { Lei regulamentares } \\
\text { específicas, Decretos etc.). }\end{array}$ & $\begin{array}{l}\text { Categórica } \\
\text { binária } \\
\text { (dummy) } \\
\text { com } \\
\text { valores "o" } \\
\text { e "1" }\end{array}$ & Nominal & $\begin{array}{c}\text { Indica se o ano do } \\
\text { desempenho observado } \\
\text { de cada escola foi antes ou } \\
\text { depois da implementação } \\
\text { do programa }\end{array}$ \\
\hline $\begin{array}{l}\text { Programa } \\
\text { (independente) }\end{array}$ & $\begin{array}{c}\text { Documentos sobre os } \\
\text { critérios oficiais para } \\
\text { provimento ao cargo } \\
\text { de diretores (PME, } \\
\text { Lei regulamentares } \\
\text { específicas, decretos etc.). }\end{array}$ & $\begin{array}{l}\text { Categórica } \\
\text { binária } \\
\text { (dummy) } \\
\text { com } \\
\text { valores "o" } \\
\text { e "1" }\end{array}$ & Nominal & $\begin{array}{l}\text { Indica se a escola faz parte } \\
\text { do grupo de controle } \\
\text { (“o” para indicações) ou } \\
\text { de tratamento (“1” para } \\
\text { eleição ou modelo misto) }\end{array}$ \\
\hline $\begin{array}{l}\text { Programa_ano } \\
\text { (independente) }\end{array}$ & $\begin{array}{l}\text { A partir dos valores } \\
\text { registrados nos bancos } \\
\text { analisados para as duas } \\
\text { variáveis anteriores }\end{array}$ & $\begin{array}{l}\text { Categórica } \\
\text { binária } \\
\text { (dummy) } \\
\text { com } \\
\text { valores "o" } \\
\text { e "1" }\end{array}$ & Nominal & $\begin{array}{c}\text { Termo interativo entre } \\
\text { as variáveis Programa e } \\
\text { Ano: só poderá apresentar } \\
\text { o valor “1” para unidades } \\
\text { do grupo de tratamento } \\
\text { e após a aplicação do } \\
\text { programa }\end{array}$ \\
\hline $\begin{array}{l}\text { Desemp_ } \\
\text { Escola_L.Port } \\
\text { (dependente) }\end{array}$ & $\begin{array}{l}\text { Microdados da Prova } \\
\text { Brasil entre os anos de } \\
2007 \text { e } 2015 \text { (Inep) }\end{array}$ & Contínua & Intervalar & $\begin{array}{c}\text { Notas das escolas em } \\
\text { Língua Portuguesa } \\
\text { relativas aos alunos } \\
\text { de } 4^{\mathrm{a}} \text { série } / 5^{\circ} \text { ano que } \\
\text { participaram da Prova } \\
\text { Brasil entre } 2007 \text { e } 2015\end{array}$ \\
\hline $\begin{array}{l}\text { Desemp_ } \\
\text { Escola_Mat } \\
\text { (dependente) }\end{array}$ & $\begin{array}{l}\text { Microdados da Prova } \\
\text { Brasil entre os anos de } \\
2007 \text { e } 2015 \text { (Inep) }\end{array}$ & Contínua & Intervalar & $\begin{array}{c}\text { Notas das escolas em } \\
\text { Matemática relativas aos } \\
\text { alunos de } 4^{\mathrm{a}} \text { série } / 5^{\mathrm{o}} \text { ano } \\
\text { que participaram da Prova } \\
\text { Brasil entre } 2007 \text { e } 2015\end{array}$ \\
\hline
\end{tabular}

Continua 
Continuação

\begin{tabular}{|l|c|l|l|l|}
\hline Lideranca & $\begin{array}{c}\text { Microdados da Prova } \\
\text { Brasil entre os anos de } \\
2007 \text { e } 2015 \text { (Inep) } \\
\text { (dependente) }\end{array}$ & Contínua & Intervalar & $\begin{array}{c}\text { Índices de liderança } \\
\text { obtidos através de Análise } \\
\text { Fatorial das respostas } \\
\text { dos professores aos } \\
\text { questionários contextuais } \\
\text { da Prova Brasil aplicados } \\
\text { entre 2007 e 2015 } \\
\text { reduzidas a um fator e com } \\
\text { agregação desses índices } \\
\text { ao nível das escolas }\end{array}$ \\
\hline $\begin{array}{l}\text { Complexidade_ } \\
\text { da_gestao } \\
\text { (não incluída } \\
\text { nas regressões. } \\
\begin{array}{l}\text { Usada para } \\
\text { análise de viés) }\end{array}\end{array}$ & $\begin{array}{c}\text { Site do Inep (http:// } \\
\text { idebescola.inep.gov.br/ } \\
\text { ideb/consulta-publica) }\end{array}$ & Discreta & Ordinal & $\begin{array}{l}\text { Indicador de complexidade } \\
\text { da gestão segundo Inep - } \\
\text { 2017/2018 }\end{array}$ \\
\hline
\end{tabular}

PME: Plano Municipal de Educação; Inep: Instituto Nacional de Estudos e Pesquisas Educacionais Anísio Teixeira.

O conteúdo de cada instrumento legal disponível foi a base para a escolha das redes, bem como a comparação entre esses instrumentos dentro de um mesmo município. Cidades com legislação relativamente clara sobre o tema, com leis que não se contradizem e/ou não geram dúvidas e, ao mesmo tempo, de alguma forma confirmam as indicações para os cargos de diretor pelo menos até o ano de 2013, tiveram suas redes municipais de ensino (e parte de suas escolas) selecionadas para o grupo de controle. Foram selecionadas redes e escolas para o grupo de tratamento a partir de municípios que possuíam as mesmas características, exceto pelo fato de terem registrado a mudança de indicação para eleição ou para modelo misto (seleção + eleição), uma única vez, também de forma clara e entre os anos de 2008 e $2013^{3}$. Após a seleção das cidades/redes públicas municipais de ensino com base nesses critérios, a listagem das cidades separadas por grupos, segue no Quadro 2.

${ }^{2}$ Alternativa semelhante foi utilizada em outros trabalhos como de Oliveira (2015), Oliveira e Waldhelm (2016) e Oliveira e Carvalho (2018).

${ }^{3}$ Para garantir a observação de pré-teste e pós-teste, considerando o período total de observação dos dados (2007 a 2015). 
Quadro 2. Organização das cidades/redes por grupos.

\begin{tabular}{|l|c|}
\hline Cidades/redes selecionadas para os grupos de controle & \\
\hline Angra dos Reis - Araruama - Areal - Belford Roxo - Bom Jardim - Cachoeiras de & \\
Macacu - Cambuci - Campos dos Goytacazes - Carapebus - Comendador Levy & \\
Gasparian - Conceição de Macabu - Duas Barras - Engenheiro Paulo de Frontin - & \\
Guapimirim - Iguaba Grande - Itaboraí - Italva - Itatiaia - Japeri - Macuco - Maricá & \\
- Mesquita - Miguel Pereira - Miracema - Nilópolis - Nova Iguaçu - Paraíba do Sul & 47 \\
- Paraty - Paty do Alferes - Pinheiral - Piraí - Porto Real - Rio das Flores - Santa & \\
Maria Madalena - Santo Antônio de Pádua - São José de Ubá - São José do Vale do & \\
Rio Preto - São Pedro da Aldeia - São Sebastião do Alto - Sapucaia - Saquarema - & \\
Silva Jardim - Sumidouro - Tanguá - Trajano de Morais - Três Rios - Varre-Sai & \\
\hline Cidades/redes selecionadas para os grupos de tratamento (eleição ou modelo misto) & \\
\hline Macaé - Niterói - São João de Meriti - Valença - Volta Redonda & 5 \\
\hline
\end{tabular}

Para cada rede que faz parte do grupo de tratamento, procurou-se captar qual o ano corresponde à mudança da forma de acesso ao cargo de diretores (saindo da indicação para eleição ou para modelo misto) a partir da legislação analisada. Tais informações estão resumidas no Quadro 3.

Quadro 3. Ano da mudança para cada cidade/rede selecionada.

\begin{tabular}{|l|c|c|c|}
\hline $\mathrm{N}^{\circ}$ & Cidade/rede & Programa & Ano da mudança \\
\hline 1 & Macaé & Seleção + eleição & 2011 \\
2 & Niterói & Eleição & 2008 \\
3 & São João de Meriti & Eleição & 2010 \\
4 & Valença & Eleição & 2010 \\
5 & Volta Redonda & Eleição & 2010 \\
\hline
\end{tabular}

Para seleção das escolas, primeiramente foram definidos três grupos de bancos de dados tanto em função das redes e escolas selecionadas para compor os grupos de controle e tratamento quanto por conta do estimador escolhido partindo da opção mais elementar de uso deste: considerando apenas uma mudança na forma de provimento ao cargo de diretores, dentro do período de 2008 e 2013 em cada banco analisado, conforme resumo apresentado no Gráfico. Para cada uma desses três conjuntos estabeleceu-se uma subdivisão em quatro bancos, baseados inicialmente em duas informações de cada escola (respostas dos diretores sobre as formas de provimento aos seus cargos e resultados de desempenho dos alunos da $4^{\mathrm{a}}$ série $/ 5^{\mathrm{o}}$ ano entre 2007 e 2015). A ideia foi elaborar desenhos mais variados para organização dos bancos que não se limitassem à legislação de cada município, principalmente pelas 
fragilidades dos dados secundários ${ }^{4}$. Assim, partiu-se de bancos com critérios de seleção de escolas mais permissivos (com escolas que apresentaram resultados de desempenho em pelo menos três edições da Prova Brasil entre 2007 e 2015, independente das respostas dos diretores sobre formas de provimento ao cargo) para processos mais restritivos (com escolas que apresentaram resultados de desempenho em todas as edições da Prova Brasil entre 2007 e 2015 e com respostas dos diretores sobre as formas de provimento ao cargo correspondentes a legislação local). Considerando o limite mínimo de 30 casos para o número de escolas por grupo, seguem os bancos que restaram para as análises (Tabela 1).

Tabela 1. Número de municípios/escolas analisadas por banco e critério de seleção.

\begin{tabular}{lcccc}
\hline Grupos de análises & Bancos/critérios & $\begin{array}{c}\text { Municípios/escolas } \\
\text { de controle }\end{array}$ & $\begin{array}{c}\text { Municípios/escolas } \\
\text { tratadas }\end{array}$ & Totais \\
\hline Grupo $^{\circ}$ 1 & Bancos com critério 1 & $47 / 605$ & $1 / 35$ & $48 / 640$ \\
\hline Grupo $^{\circ}$ 2 & Bancos com critério 1 & $47 / 605$ & $1 / 34$ & $48 / 649$ \\
\hline \multirow{2}{*}{ Grupo n $^{\circ} 3$} & Bancos com critério 1 & $47 / 605$ & $3 / 93$ & $50 / 701$ \\
& Bancos com critério 2 & $43 / 330$ & $3 / 57$ & $46 / 387$ \\
\hline
\end{tabular}

Em seguida foram avaliados padrões e mecanismos de não respostas em cada banco, além dos limites e possibilidades de diferentes técnicas de imputação de dados, decidindo-se pelo uso da Imputação Múltipla ${ }^{5}$. Como método neste caso, recorreu-se ao procedimento conhecido como Monte Carlo via Cadeias de Markov (Markov Chain Monte Carlo - MCMC) que se trata de um método linear normal multivariado. Este método poder lidar com praticamente todo tipo de padrão de perda de casos, além de sua eficiência em programas convencionais, como o SPSS (utilizado para as análises).

Realizaram-se comparações entre os diferentes bancos, bem como dentro de cada banco (comparando-se grupos de controle e tratamento) para analisar possíveis vieses de seleção em relação à complexidade de gestão das escolas. A intenção foi perceber nessas comparações se entre os grupos de escolas selecionadas havia diferenças estatisticamente significativas nas frequências dos níveis de complexidade de gestão, o que poderia indicar vieses de seleção. Isto porque, na medida em que existam diferenças significativas entre essas frequências, pode haver também uma variação importante entre os grupos no nível de dificuldade em gerir as respectivas escolas, independente das

\footnotetext{
${ }^{4}$ Ver discussão correspondente em Cunha (ibidem).

${ }^{5}$ O trabalho de Cunha (2019) apresenta a técnica de Imputação Múltipla de forma detalhada incluindo explanações sobre padrões em mecanismos de não resposta, o passo a passo para a aplicação deste recurso e diversos apêndices com tabelas apresentando os dados gerais de todas as imputações realizadas na pesquisa.
} 
características dos diretores. Foram realizados testes Qui-quadrados de independência, para a comparação de frequências. Não foram constatados vieses de seleção que comprometessem as análises considerando as comparações sugeridas. Em relação à Análise Fatorial para a criação dos índices de liderança dos diretores por escola, as Tabelas 2 e 3 apresentam os valores das cargas fatoriais para as variáveis correspondentes ${ }^{6}$.

Tabela 2. Cargas fatoriais das variáveis relativas à liderança dos diretores (banco com $1^{\circ}$ critério).

\begin{tabular}{lc}
\hline Índice de liderança do diretor com seis componentes & Carga \\
\hline Variável & Fatorial \\
\hline O(A) diretor(a) me anima e me motiva para o trabalho & 0,873 \\
Tenho plena confiança no(a) diretor(a) como profissional & 0,823 \\
$\mathrm{O}(\mathrm{A})$ diretor(a) estimula atividades inovadoras & 0,866 \\
$\mathrm{O}(\mathrm{A})$ diretor(a) dá atenção especial a aspectos relacionados com a aprendizagem dos alunos & 0,843 \\
Sinto-me respeitado(a) pelo(a) diretor(a) & 0,768 \\
O diretor, professores e demais membros da equipe da escola colaboram para fazer esta & 0,733 \\
\hline
\end{tabular}

Fonte: Microdados Inep. Elaboração própria.

Tabela 3. Cargas fatoriais das variáveis relativas à liderança dos diretores (banco com $2^{\circ}$ critério).

\begin{tabular}{lc}
\hline Índice de liderança do diretor com seis componentes & \\
\hline Variável & Carga \\
& Fatorial \\
\hline O(A) diretor(a) me anima e me motiva para o trabalho & 0,870 \\
Tenho plena confiança no(a) diretor(a) como profissional & 0,817 \\
$\mathrm{O}(\mathrm{A})$ diretor(a) estimula atividades inovadoras & 0,865 \\
$\mathrm{O}(\mathrm{A})$ diretor(a) dá atenção especial a aspectos relacionados com a aprendizagem dos alunos & 0,842 \\
Sinto-me respeitado(a) pelo(a) diretor(a) & 0,767 \\
O diretor, professores e demais membros da equipe da escola colaboram para fazer esta & 0,725 \\
escola funcionar bem demais & \\
\hline
\end{tabular}

Fonte: Microdados Inep. Elaboração própria.

Todas as cargas fatoriais observadas são significativas. Stevens (1992 apud. FIELD, 2009) recomenda o valor mínimo de 0,722 para uma carga fatorial ser considerada significativa para amostras com pelo menos 50 unidades. Todas as nossas amostras analisadas são bem maiores e a carga fatorial mínima obtida acima foi de 0,725.

${ }^{6}$ Em Cunha (ibidem) há maior detalhamento sobre a escolha dessas variáveis para a criação do índice. 
Destarte, partimos para o método Diferença em Diferenças/diff-in-diff (MAFFIOLI, 201 1; ALBOUY, 2004; ABADIE, 2003; FOGUEL, 2012), que tem a capacidade de controlar características não observáveis entre os grupos comparados. Optamos pela sua aplicação através de regressões lineares (GREMAUD, PAZELLO, MALUF, 2016; SANTOS, SAMPAIO, SAMPAIO, 2015; FOGUEL, 2012). Assim, tendo como forma geral:

$$
\bar{Y} \text { it }=\alpha+\beta 1 \text { Programa }_{\mathrm{it}}+\beta 2 \text { Ano }_{\mathrm{t}}+\beta 3 \text { Programa }_{\mathrm{it}} *{\text { Anot }+\varepsilon_{\mathrm{i}}}
$$

A variável binária "Programa” indica se cada unidade de análise participou ou não do mesmo, tendo respectivamente os valores “1” e “o”. A variável “Ano” exibe valor igual a "O" quando o desempenho de uma escola foi registrado antes do tratamento (ou seja, com $t=0$ ) e "1" após o mesmo (com $t=1$ ). O mesmo esquema foi aplicado para o Índice de Liderança. A variável “programa*ano” é o termo interativo entre as duas variáveis anteriores e só poderá apresentar o valor “1” para unidades do grupo de tratamento e após a aplicação do programa. Já o coeficiente $\beta 3$, por estar associado à interação, é justamente o efeito do tratamento na variável dependente a ser observada.

\section{Resultados e discussão}

Análise diff-in-diff com as escolas de Niterói

Comparando-se os dois grupos, sendo o de tratamento com as 35 escolas de Niterói, apresenta-se (a título de explicação do mecanismo utilizado e como exemplo) a Tabela 4 que exibe o cálculo da diferença, ao longo do tempo (considerando as diferenças das médias de proficiência em Matemática). Registra-se assim o efeito da adoção de eleição para diretores nas escolas niteroienses selecionadas com base na comparação entre 2007 e 2009 para depois ser aplicado o estimador. Para as demais variáveis e cidades/escolas analisadas, seguem apresentadas somente as tabelas referentes à aplicação do diff-in-diff.

Tabela 4. Cálculo da diferença em diferenças entre o grupo de tratamento (Niterói) e controle para média em Matemática (2007 a 2009).

\begin{tabular}{lccc}
\hline & $\begin{array}{c}\text { Antes do } \\
\text { tratamento }\end{array}$ & $\begin{array}{c}\text { Depois do } \\
\text { tratamento }\end{array}$ & $\begin{array}{c}\text { Mudança nas } \\
\text { proficiências }\end{array}$ \\
\cline { 2 - 4 } & Ano de 2007 & Ano de 2009 & \\
\hline Escolas do grupo de tratamento & 188,24 & 200,47 & 12,23 \\
Escolas do grupo de controle & 195,93 & 198,56 & 2,63 \\
\hline Diferença média na proficiência em Matemática & $-7,69$ & 1,90 & 9,60 \\
\hline
\end{tabular}

Fonte: Microdados Inep. Elaboração própria. 
A Tabela 4 (assim como as demais produzidas para o estudo) sugere efeitos positivos tanto em Matemática quanto nas demais variáveis dependentes após a adoção de eleições em Niterói. Entretanto, para sabermos se tais mudanças são estatisticamente significativas, necessitamos aplicar o diff-in-diff por meio de regressões lineares. Os resultados seguem apresentados nas Tabelas 5 a 7 .

Tabela 5. Impacto da política de eleição de diretores sobre a proficiência média em Matemática em escolas municipais de Niterói (2007 a 2009): estimativas por diferença em diferenças.

\begin{tabular}{lcc}
\hline & Modelo 1 & Modelo 2 \\
\hline Política (dummy) & $-0,9502$ & $-7,2940^{*}$ \\
& $(1,6141)$ & $(3,6077)$ \\
Ano $_{2009}($ dummy) & $8,9412^{* * *}$ & $8,5073^{* * *}$ \\
& $(0,9178)$ & $(0,9436)$ \\
Interação (Política*Ano $\left.{ }_{2009}\right)$ & - & $7,9298^{*}$ \\
$\mathrm{R}^{2}$ & - & $(4,0335)$ \\
\hline Controles & 0,0291 & 0,0287 \\
\hline Número de observações & Não & Não \\
\hline
\end{tabular}

Fonte: Microdados Inep. Elaboração própria.

* Significativo ao nível de 10\%; *** Significativo ao nível de $1 \%$.

Tabela 6. Impacto da política de eleição de diretores sobre a proficiência média em Língua Portuguesa em escolas municipais de Niterói (2007 a 2009): estimativas por diferença em diferenças

\begin{tabular}{lcc}
\hline & Modelo 1 & Modelo 2 \\
\hline Política (dummy) & 0,7152 & $-4,9584$ \\
& $(1,3425)$ & $(3,0003)$ \\
$\mathrm{Ano}_{2009}($ dummy) & $10,909^{* * * *}$ & $10,5207^{* * * *}$ \\
& $(0,7634)$ & $(0,7847)$ \\
Interação (Política*Ano $\left.{ }_{2009}\right)$ & - & $7,0919 * *$ \\
$\mathrm{R}^{2}$ & - & $(3,3545)$ \\
Controles & 0,0601 & 0,0650 \\
\hline Número de observações & Não & Não \\
\hline
\end{tabular}

Fonte: Microdados INEP. Elaboração própria.

** Significativo ao nível de $5 \%$; *** Significativo ao nível de $1 \%$. 
Tabela 7. Impacto da política de eleição de diretores sobre o Índice de liderança em escolas municipais de Niterói (2007 a 2009): estimativas por diferença em diferenças.

\begin{tabular}{lcc}
\hline & Modelo 1 & Modelo 2 \\
\hline Política (dummy) & 0,0584 & 0,0110 \\
& $(0,0647)$ & $(0,1446)$ \\
$\mathrm{Ano}_{2009}($ dummy) & $-0,0507$ & $-0,0539$ \\
& $(0,0368)$ & $(0,0378)$ \\
Interação (Política*Ano $\left.{ }_{2009}\right)$ & - & 0,0593 \\
$\mathrm{R}^{2}$ & - & $(0,1617)$ \\
\hline Controles & 0,0009 & 0,0005 \\
\hline Número de observações & Não & Não \\
\hline
\end{tabular}

Fonte: Microdados Inep. Elaboração própria.

As primeiras colunas das três tabelas anteriores e das demais desta seção (referentes ao uso do estimador) exibem os resultados dos modelos os quais utilizamos duas variáveis independentes: "Programa” e “Ano”. Já na segunda coluna de cada uma dessas mesmas tabelas, os modelos foram aplicados com a inclusão do termo interativo ('Programa*Ano'). Devido ao caráter exploratório do estudo, em nenhum dos dois modelos fizemos a inclusão de outros controles. Ainda assim, nota-se que o termo interativo (justamente o efeito da política) é significativo para a média de proficiência em Matemática (significância a 10\%) e Língua Portuguesa ( ignificância a 5\%) nas escolas de Niterói. O mesmo não ocorreu com Índice de liderança dos diretores. Na comparação com os dois modelos, temos efeito nas variáveis dependentes de desempenho (com significância a 1\%) a partir da variável independente "Ano”, sendo este efeito pouco relevante para o estudo, primeiramente porque o foco está no impacto da política e segundo porque percebe-se nas demais análises que quase sempre esta variável tem impacto significativo pela simples tendência (inclusive em nível nacional) de aumento dos escores das escolas com o passar do tempo, podendo ocorrer por distintos fatores, alheios ao que discute-se neste trabalho. Como para os demais bancos foram realizadas análises de maneira análoga, adiante apresentam-se apenas as tabelas referentes à aplicação do estimador, com os respectivos resultados:

Análise diff-in-diff com as escolas de Macaé

As Tabelas 8 a 10 mostram os resultados para o município de Macaé. 
Tabela 8. Impacto da política de seleção + eleição de diretores sobre a proficiência média em Matemática em escolas municipais de Macaé (2011 a 2013): estimativas por diferença em diferenças.

\begin{tabular}{lcc}
\hline & Modelo 1 (sem o termo interativo) & Modelo 2 (com o termo interativo) \\
\hline Política (dummy) & $16,471^{* * *}$ & $18,245^{* * *}$ \\
& $(1,645)$ & $(1,952)$ \\
$\mathrm{Ano}_{2009}($ dummy) & $7,048^{* * * *}$ & $7,382^{* * * *}$ \\
\multirow{2}{*}{ Interação (Política* Ano $\left.{ }_{2013}\right)$} & $(0,748)$ & $(0,772)$ \\
& - & $-4,531$ \\
$\mathrm{R}^{2}$ & - & $(2,672)$ \\
\hline Controles & 0,056 & 0,057 \\
\hline Número de observações & Não & Não \\
\hline
\end{tabular}

Fonte: Microdados Inep. Elaboração própria.

*** Significativo ao nível de $1 \%$.

Tabela 9. Impacto da política de seleção + eleição de diretores sobre a proficiência média em Língua Portuguesa em escolas municipais de Macaé (2011 a 2013): estimativas por diferença em diferenças.

\begin{tabular}{lcc}
\hline & Modelo 1 (sem o termo interativo) & Modelo 2 (com o termo interativo) \\
\hline Política (dummy) & $16,015^{* * * *}$ & $17,471^{* * *}$ \\
& $(1,330)$ & $(1,577)$ \\
$\mathrm{Ano}_{2009}($ dummy) & $12,124^{* * * *}$ & $12,404^{* * *}$ \\
& $(0,605)$ & $(0,624)$ \\
Interação (Política* Ano $\left._{2013}\right)$ & - & $-3,723$ \\
& - & $(2,158)$ \\
$\mathrm{R}^{2}$ & 0,146 & 0,147 \\
\hline Controles & $\mathrm{Não}$ & $\mathrm{Não}$ \\
\hline Número de observações & 639 & 639 \\
\hline
\end{tabular}

Fonte: Microdados Inep. Elaboração própria.

*** Significativo ao nível de $1 \%$.

Tabela 10. Impacto da política de seleção + eleição de diretores sobre o Índice de liderança em escolas municipais de Macaé (2011 a 2013): estimativas por diferença em diferenças.

\begin{tabular}{lcc}
\hline & Modelo 1 (sem o termo interativo) & Modelo 2 (com o termo interativo) \\
\hline Política (dummy) & 0,038 & 0,055 \\
& $(0,065)$ & $(0,077)$ \\
Ano $_{2009}($ dummy) & $-0,299^{*} * *$ & $-0,296^{* * * *}$ \\
& $(0,029)$ & $(0,030)$ \\
Interação (Política* Ano $\left._{2013}\right)$ & - & $-0,044$ \\
& - & $(0,105)$ \\
$\mathrm{R}^{2}$ & 0,032 & 0,032 \\
\hline Controles & Não & Não \\
\hline Número de observações & 639 & 639 \\
\hline
\end{tabular}

Fonte: Microdados Inep. Elaboração própria.

*** Significativo ao nível de $1 \%$. 
Nenhuma das análises com as escolas de Macaé exibiu impactos significativos da política de escolha de diretores via modelo misto, ao menos sem a inclusão de outros controles. Apenas a variável “Ano” aparece (novamente) com valores estatisticamente significativos.

Análise diff-in-diff com as escolas de São João de Meriti, Valença e Volta Redonda (1 ${ }^{\circ}$ critério)

Os resultados estatísticos das análises seguem nas Tabelas 11 a 13 .

Tabela 11. Impacto da política de eleição de diretores sobre a proficiência média em Matemática em escolas municipais dos demais municípios/ $1^{\circ}$ critério (2009 a 2011): estimativas por diferença em diferenças.

\begin{tabular}{lcc}
\hline & Modelo 1 (sem o termo interativo) & Modelo 2 (com o termo interativo) \\
\hline Política (dummy) & $5,940^{* * * *}$ & 2,711 \\
& $(1,039)$ & $(1,642)$ \\
Ano $_{2009}($ dummy) & $9,276^{* * *}$ & $8,559^{* * * *}$ \\
& $(0,721)$ & $(0,774)$ \\
Interação (Política*Ano $\left.{ }_{2011}\right)$ & - & $5,378^{* * *}$ \\
& - & $(2,120)$ \\
$\mathrm{R}^{2}$ & 0,054 & 0,056 \\
\hline Controles & Não & Não \\
\hline Número de observações & 698 & 698 \\
\hline
\end{tabular}

Fonte: Microdados Inep. Elaboração própria.

*** Significativo ao nível de $1 \%$.

Tabela 12. Impacto da política de eleição de diretores sobre a proficiência média em Língua Portuguesa em escolas municipais dos demais municípios $/ 1^{\text {o }}$ critério (2009 a 2011): estimativas por diferença em diferenças.

\begin{tabular}{lcc}
\hline & Modelo 1 (sem o termo interativo) & Modelo 2 (com o termo interativo) \\
\hline Política (dummy) & $6,108^{* * *}$ & 2,897 \\
& $(0,858)$ & $(1,355)$ \\
Ano $_{2009}($ dummy) & $11,820^{* * *}$ & $11,106^{* * *}$ \\
& $(0,596)$ & $(0,639)$ \\
Interação (Política* Ano $\left._{2011}\right)$ & - & $5,350^{* * *}$ \\
& - & $(1,749)$ \\
$\mathrm{R}^{2}$ & 0,113 & 0,116 \\
\hline Controles & Não & Não \\
\hline Número de observações & 698 & 698 \\
\hline
\end{tabular}

Fonte: Microdados Inep. Elaboração própria.

*** Significativo ao nível de $1 \%$. 
Tabela 13. Impacto da política de eleição de diretores sobre o Índice de liderança em escolas municipais dos demais municípios $/ 1^{\circ}$ critério (2009 a 2011): estimativas por diferença em diferenças.

\begin{tabular}{lcc}
\hline & Modelo 1 (sem o termo interativo) & Modelo 2 (com o termo interativo) \\
\hline Política (dummy) & 0,101 & 0,097 \\
& $(0,041)$ & $(0,065)$ \\
$\mathrm{Ano}_{2009}($ dummy) & $-0,150^{* * * *}$ & $-0,151^{* * *}$ \\
& $(0,028)$ & $(0,031)$ \\
Interação (Política*Ano $\left.{ }_{2011}\right)$ & - & 0,007 \\
& - & $(0,084)$ \\
$\mathrm{R}^{2}$ & 0,010 & 0,010 \\
\hline Controles & $\mathrm{Não}$ & $\mathrm{Não}$ \\
\hline Número de observações & 698 & 698 \\
\hline
\end{tabular}

Fonte: Microdados Inep. Elaboração própria.

*** Significativo ao nível de $1 \%$.

Tanto em Matemática quanto em Língua Portuguesa, a adoção das novas políticas de escolha de diretores nas escolas selecionadas nestas três cidades, tiveram impactos significativos (a 5\% e 1\%, respectivamente). Em Matemática e Língua Portuguesa, o efeito é significativo e positivo. A variável "Ano”, como esperado, mais uma vez também apresentou significância (a 1\%). Uma curiosidade é que agora a variável "Política” aparece significativa para as variáveis de desempenho e nos dois modelos aplicados (com e sem o termo interativo). É um indicativo de que há diferenças estatisticamente significativas entre as 93 escolas tratadas nas três cidades em questão e as demais escolas de controle, independentemente da adoção das eleições.

Análise diff-in-diff com as escolas de São João de Meriti, Valença e Volta Redonda (2º critério)

As Tabelas 14 a 16 apresentam os resultados do diff-in-diff e as respectivas discussões.

Tabela 14. Impacto da política de eleição de diretores sobre a proficiência média em Matemática em escolas municipais dos demais municípios/2 ${ }^{\circ}$ critério (2009 a 2011 ): estimativas por diferença em diferenças.

\begin{tabular}{lcc}
\hline & Modelo 1 (sem o termo interativo) & Modelo 2 (com o termo interativo) \\
\hline Política (dummy) & $11,137^{* * * *}$ & $7,339^{* * * *}$ \\
& $(1,208)$ & $(1,908)$ \\
Ano $_{2009}($ dummy) & $9,243^{* * *}$ & $8,367^{* * *}$ \\
& $(0,849)$ & $(0,914)$ \\
Interação (Política*Ano $\left.{ }_{2011}\right)$ & - & $6,328^{* *}$ \\
& - & $(2,463)$ \\
$\mathrm{R}^{2}$ & 0,088 & 0,091 \\
\hline Controles & Não & Não \\
\hline Número de observações & 387 & 387 \\
\hline
\end{tabular}


Tabela 15. Impacto da política de eleição de diretores sobre a proficiência média em Língua Portuguesa em escolas municipais dos demais municípios/2o critério (2009 a 2011): estimativas por diferença em diferenças.

\begin{tabular}{lcc}
\hline & \multicolumn{2}{c}{ Proficiência em Língua Portuguesa } \\
\cline { 2 - 3 } & Modelo 1 (sem o termo interativo) & Modelo 2 (com o termo interativo) \\
\hline Política $($ dummy) & $10,335^{* * * *}$ & $6,078^{* * * *}$ \\
& $(1,091)$ & $(1,722)$ \\
Ano $_{2009}($ dummy) & $11,864^{* * * *}$ & $10,885^{* * *}$ \\
& $(0,768)$ & $(0,826)$ \\
Interação (Política*Ano & & \\
& - & $7,092^{* * * *}$ \\
$\mathrm{R}^{2}$ & - & $(2,223)$ \\
Controles & 0,135 & 0,139 \\
\hline Número de observações & Não & Não \\
\hline
\end{tabular}

Fonte: Microdados Inep. Elaboração própria.

*** Significativo ao nível de $1 \%$.

Tabela 16. Impacto da política de eleição de diretores sobre o Índice de liderança em escolas municipais dos demais municípios $/ 2^{\circ}$ critério (2009 a 2011): estimativas por diferença em diferenças.

\begin{tabular}{lcc}
\hline & \multicolumn{2}{c}{ Índice de liderança } \\
\cline { 2 - 3 } & Modelo 1 (sem o termo interativo) & Modelo 2 (com o termo interativo) \\
\hline Política (dummy) & $0,156^{* * * *}$ & 0,142 \\
& $(0,051)$ & $(0,081)$ \\
Ano $_{2009}$ (dummy) & $-0,157^{* * *}$ & $-0,160^{* * * *}$ \\
& $(0,036)$ & $(0,039)$ \\
Interação (Política*Ano $\left.{ }_{2011}\right)$ & - & 0,023 \\
& - & $(0,104)$ \\
$\mathrm{R}^{2}$ & 0,014 & 0,014 \\
\hline Controles & Não & Não \\
\hline Número de observações & 387 & 387 \\
\hline
\end{tabular}

Fonte: Microdados Inep. Elaboração própria.

*** Significativo ao nível de $1 \%$.

As variáveis "Ano" e "Política” são significativas a 1\%, nas três variáveis e nos dois modelos utilizados (exceto "Política" no segundo modelo - com o termo interativo - para a variável "Liderança”). De modo geral, tanto o tempo, quanto o fato de fazer parte do grupo de controle ou tratamento gerou diferenças estatisticamente significativas nos resultados. Contudo o termo interativo aqui também tem o seu espaço: 
foi significativo em Matemática e Língua Portuguesa a 5\% e 1\%, respectivamente, ou seja, para além da mudança com o tempo em ambos os grupos ou da anterior diferença entre eles, a adoção das eleições a partir de 2010, também gerou efeitos.

\section{Considerações finais}

Houve um aumento da proficiência média das escolas tanto Língua Portuguesa quanto em Matemática ao longo dos anos. Esta é uma tendência observada inclusive em outras regiões e no cenário nacional como um todo. Tal variação pode ocorrer por fatores distintos para além do que estamos discutindo neste trabalho.

Em nenhuma das análises a variável "liderança” apresentou resultados estatisticamente significativos. Porém, considerando tendências, percebe-se que, na maioria das análises, os resultados sugerem efeitos positivos das eleições nos índices de liderança com exceção das escolas de Macaé, após a adoção do modelo misto. Oliveira e Waldhelm $(2016)^{7}$ e Oliveira e Carvalho (2018), ao estudarem a percepção de Liderança dos diretores por parte dos docentes e a sua relação com desempenho dos estudantes, nos trazem algumas pistas. Estes estudos registraram percepções negativas sobre liderança dos diretores por parte dos professores, tal qual também foi constatado no presente estudo através das estatísticas descritivas de todos os grupos, principalmente a partir de 2011 - nos estudos de Oliveira e Carvalho (2018) a queda maior foi em 2009. A hipótese (a ser investigada) é a de que “a difusão crescente de políticas de responsabilização nos últimos anos no Brasil [...]pode estar influenciando a percepção dos professores sobre a liderança do diretor e aumentando a atenção deste sobre os resultados de aprendizagem dos alunos" (ibidem, p. 12 e 13). Podemos inferir que possivelmente parte dos professores passou a ser mais criteriosa nas avaliações de seus diretores e/ou determinadas expectativas que se tinha sobre esses últimos não foram correspondidas. Também se nota o efeito negativo do processo de seleção + eleição de diretores no caso do desempenho em Matemática, novamente nas escolas tratadas em Macaé. Nesta cidade as mudanças ocorreram no ano de 2012 com a posse dos novos diretores eleitos sendo que em boa parte das escolas os diretores antigos foram reconduzidos. Algo que pode explicar, em parte, o fato de as escolas neste município de modo geral não

\footnotetext{
${ }^{7}$ Nestas pesquisas, há uma diferença em relação ao presente estudo, pois houve a utilização de liderança do diretor como variável preditora e a sua associação positiva nos resultados acadêmicos dos alunos (sendo que na presente pesquisa considerou-se o índice de liderança como variável dependente das variações nas formas de provimento aos cargos).
} 
apresentarem mudanças estatisticamente significativas (e tendências negativas) após a adoção do modelo misto para escolha de diretores. Em todas as demais redes com escolas tratadas houve efeitos positivos e estatisticamente significativos no desempenho dos estudantes tanto em Língua Portuguesa, quanto em Matemática (sem os controles via características dos alunos, das escolas ou dos professores) e as médias dos Índices de liderança também tiveram efeitos positivos (embora não significativos), ou seja, na mesma direção com o que ocorreu com desempenho. De todas as comparações, as que contaram com o grupo de tratamento com as 58 escolas das cidades de São João de Meriti, Valença e Volta Redonda e com o grupo com as 34 escolas de Macaé apresentaram os dados com as mudanças mais marcadas e coerentes conforme esperado (ao menos nas frequências de respostas dos diretores sobre as formas de provimento). Constatou-se ainda os baixos valores de $\mathrm{R}_{2}$, mostrando que os modelos utilizados explicam pouco das variações nas médias, sendo necessária a inclusão de novas variáveis para as análises.

Para além de captar efeitos e tendências nos diferentes grupos de escolas analisados, este trabalho procurou também contribuir com a possibilidade de arranjos diferenciados para desenhos de pesquisa que não se limitem apenas o que está disposto documentos oficiais dos municípios, por exemplo.

\section{Referências}

ABADIE. A. Semiparametric difference-in-differences estimators. Review of Economic Studies, Oxford, v. 72, n. 1, p. 1-19, jan. 2005.

ALBOUY, D. Program evaluation and the difference in difference estimator. Economics, v. 131, p. 1-4, 2004.

ALVES, F. C. M. Qualidade na educação fundamental pública nas capitais brasileiras: tendências, contextos e desafios. 2007. 243 fls. Tese (Doutorado em educação) — Pontifícia Universidade Católica do Rio de Janeiro, Rio de Janeiro, RJ, 2007. Disponível em: <http://www.observatoriodasmetropoles.ufrj.br/download/tese_fatima_07.pdf >. Acesso em: 12 ago. 2019.

BIONDI, R. L.; FELÍCIO, F. Atributos escolares e o desempenho dos estudantes: uma análise em painel dos dados do SAEB. Brasília, DF: Instituto Nacional de Estudos e Pesquisas Educacionais Anísio Teixeira, 2008. Disponível em: <https://docplayer.com. br/46499-Atributos-escolares-e-o-desempenho-dos-estudantes-uma-analise-em-painel-dos-dados-do-saeb.html>. Acesso em: 15 jul. 2019. 
CAMPBELL, D. T.; STANLEY, J. C. Experimental and quasi-experimental designs for research on teaching. In: GAGE, N. L. (Ed.). Handbook of research on teaching. Chicago, IL: Rand McNally, 1963. p. 171-246

Cunha, S. G. Formas de provimento ao cargo de diretor, desempenho e liderança em escolas públicas no estado do Rio de Janeiro. 2019. Tese (Doutorado em educação) — Faculdade de Educação, Universidade Federal do Rio de Janeiro, Rio de Janeiro, RJ, 2019. Disponível em: <https://ppge.educacao.ufrj.br/teses2019/tSergio\%20Gon\%C3\%A7alves\%20da\%20Cunha.pdf >. Acesso em: 9 ago. 2019.

DHUEY, E.; SMITH, J. How important are school principals in the production of student achievement? Toronto: University of Toronto, 2011. Disponível em < http://www.sole jole.org/1 1034.pdf>. Acesso em: 26 out. 2018.

ESQUINSANI, R. S. S. Eleição de diretores e gestão da escola pública: reflexões sobre democracia e patrimonialismo. Nuances: Estudos sobre Educação, Presidente Prudente, v. 24, n. 2, p. 101-15, maio/ago. 2013.

FIELD, A. Descobrindo a estatística usando o SPSS-2. Porto Alegre, RS: Bookman, 2009.

FOGUEL, M. N. Métodos básicos de avaliação de impacto. In: MENEZES, N. (Org.). Avaliação econômica de projetos sociais. São Paulo, SP: Dinâmica, 2012. p. 31-106. Disponível em: <http://www.pucsp.br/ecopolitica/downloads/B_2012_Avaliacao_Programas_Sociais.pdf>. Acesso em: 24 jan. 2018.

GREMAUD, A. P.; PAZELLO, E. T.; MALUF, B. T. O impacto da indicação política do diretor escolar no desempenho educacional. In: Reunião da ABAVE - Avaliação de Larga Escala no Brasil: Ensinamentos, Aprendizagens e Tendências, 8, 2015, Florianópolis. Anais...Recife, PE: Associação Brasileira de Avaliação Educacional, 2016. Disponível em: <http://abave.com.br/ojs/index.php/Reunioes_da_Abave/article/ viewFile/302/103>. Acesso em: 5 ago. 2019.

LEITHWOOD, K.; JANTZI, D. Principal and teacher leader effects: a replication. School Leadership and Management, v. 20, n. 4, p. 415-34, 2000. https://doi. org/10.1080/713696963

LEITHWOOD, K.; SUN, J. Transformational school leadership effects on schools, teachers and students. In: HOY, W. K.; DIPAOLA, M. (Eds.). School improvement. New York, NY: Information Age, 2009. p. 1-22. 
LÜCK, H. Dimensões de gestão escolar e suas competências. São Paulo, SP: Positivo, 2009. Disponível em: <http://www.fundacoes.org.br/uploads/estudos/gestao_escolar/dimensoes_livro.pdf> Acesso em: 1 ago. 2019.

Mapeamento de práticas de seleção e capacitação de diretores escolares. São Paulo, SP: Fundação Victor Civita, 2011. Disponível em: <http://www.institutounibanco. org.br/wp-content/uploads/2013/07/mapeamento_de_praticas_de_selecao_e_capacitacao_de_gestores_escolares.pdf>. Acesso em: 10 ago. 2019.

MAFFIOLI, A. Non-experimental methods: propensity score matching and difference in difference. Cuernavaca: Inter-American Development Bank, 2011. Disponível em: <http://www.impactevaluation2011.org/forum/wp-content/uploads/2011/06/ C5-commissioners-of-evaluations-file- 1.pdf >. Acesso em: 16 ago. 2019.

OLIVEIRA, A. C. P. As relações entre direção, liderança e clima escolar em escolas municipais do Rio de Janeiro. 2015. Tese (Doutorado) - Departamento de Educação, Pontificia Universidade Católica do Rio de Janeiro, Rio de Janeiro, RJ, 2015. Disponível em: <http://www.maxwell.vrac.puc-rio.br/25774/25774.PDF>. Acesso em: 1 ago. 2018.

OLIVEIRA, A. C. P.; CARVALHO, C. P. Gestão escolar, liderança do diretor e resultados educacionais no Brasil. Revista Brasileira de Educação, Rio de Janeiro, v. 23, mar. 2018. https://doi.org/10.1590/s 1413-24782018230015

OLIVEIRA, A. C. P.; WALDHELM, A. P. S. Liderança do diretor, clima escolar e desempenho dos alunos: qual a relação? Ensaio: Avaliação e Políticas Públicas em Educação, Rio de Janeiro, v. 24, n. 93, p. 824-44, out./dez. 2016. https://doi.org/10.1590/ S0104-40362016000400003

PENA, A. C. Um conceito para liderança escolar. estudo realizado com diretores de escolas da rede pública estadual de Minas Gerais. 2013. 185 fls. Tese (Doutorado) — Faculdade de Educação, Universidade Federal de Juíz de Fora, Juíz de Fora, MG, 2013. Disponível em: <https://repositorio.ufjf.br/jspui/handle/ufjf/2426>. Acesso em: 23 out. 2018.

POLON, T. L. P. Identificação dos perfis de liderança e características relacionadas à gestão pedagógica eficaz nas escolas participante do projeto GERES estudo longitudinal: geração escolar 2005: pólo Rio de Janeiro. 2009. 17 fls. Tese (Doutorado em Educação) — Pontifícia Universidade Católica do Rio de Janeiro, Rio de Janeiro, RJ, 2009. Disponível em: <http://www.dbd.puc-rio.br/pergamum/tesesabertas/0510368_o9_pretextual. pdf $>$. Acesso em: 26 jul. 2018. 
SAMMONS, P. As características-chave das escolas eficazes. In: BROOKE, N.; SOARES, J. F. (Org.). Pesquisa em eficácia escolar: origem e trajetórias. Belo Horizonte, MG: Universidade Federal de Minas Gerais, 2008. p.335-92.

SANTOS, F. J. S.; SAMPAIO, R. M. B.; SAMPAIO, L. M. B. Eleição nas escolas: análise do impacto do diretor eleito sobre o desempenho educacional no estado da Bahia. In: Encontro da ANPAD, 38. 2014, Rio de Janeiro. Anais... Maringá, PR: Associação Nacional de Pós-Graduação e Pesquisa em Administração, 2014. Disponível em: $<$ http://www.anpad.org.br/admin/pdf/2014_EnANPAD_APB1937.pdf>. Acesso em: 4 mar. 2019.

Submissão em: 09/10/2019

Aceito em: 14/10/2019 